\title{
Comparative health status of patients with 11 common illnesses in Wales
}

\author{
Ronan A Lyons, Su Vui Lo, Beverley N C Littlepage
}

\begin{abstract}
Objective - To assess the health status of patients with 11 common illnesses asthma, diabetes, arthritis, back pain, sciatica, hypertension, angina, anxiety, depression, and heart attack and stroke.

Design - Face to face interview using a structured questionnaire which contained the Short Form 36 questionnaire (SF-36) and questions on lifestyle, health service utilisation, and self reported conditions treated by physicians.

Setting - Patients' homes, in West Glamorgan, Wales.

Subjects - Twelve hundred adults, aged 20-89 years, were randomly selected from the register of the family health services authority.

Main outcome measures - The eight scales within the SF-36 health profile.

Results - The response rate was $82 \%$. Each illness had a distinctive profile; patients with anxiety or depression reported the worst health experience in role limitations because of emotional problems and mental health, while patients with back pain, arthritis, or sciatica registered the three highest negative scores in bodily pain and role limitations due to physical problems. For all disease groups, the general health perceptions of those with the disease was significantly worse than those without it $(p<0.01)$.

Conclusions - The SF-36 allows comparison of the health status of patients suffering from different conditions. Data such as these can be used to inform better purchasing decisions on how resources might be more effectively deployed and as a bench mark to monitor the effects of multiple health care interventions by conducting serial surveys.
\end{abstract}

\section{( $\mathcal{F}$ Epidemiol Community Health 1994;48:388-390)}

Measures of health status and outcome centered on patients have recently been recognised as being of vital importance for monitoring quality and achieving cost effectiveness of health care services. A generic health status measuring tool, the Short Form 36 questionnaire (SF-36), developed for use in the Medical Outcomes Study in America, ${ }^{12}$ has recently shown much promise in delivering information on patient outcomes. This instrument has been anglicised and validated for use in Britain in two population based postal surveys. ${ }^{34}$

The SF-36 measures factors of prime concern to the patients, namely functional status, well being, and overall health. It provides yardsticks for health status comparisons both in subjects with the same condition and between those with different conditions. This paper reports the findings of a health status survey involving a random sample of the general population, in which the health profiles of patient groups suffering from a variety of common medical conditions are presented.

\section{Methods}

In March 1993, a questionnaire which contained the SF-36 and a variety of questions on lifestyle, health service utilisation, and self reported physician treated conditions was administered by trained health visitors to a sample of adults, aged 20-89 years, from West Glamorgan in their own homes. The study population was selected by taking a systematic sample of 1200 people (every 310th patient) from the family health services authority register. The sample size required was calculated with a power of $80 \%$ to detect a $5 \%$ point change in physical functioning in serial survey at a significance level of $1 \%$. The calculation indicated that a sample size of 842 completed interviews would be required, and the final sample of 1200 was selected on the basis of allowing for a degree of inaccuracy in the register and an estimated response rate of $85 \%$.

People who had died or moved away were identified by the interviewers questioning the new inhabitants, relatives, or neighbours during household interviews after a minimum of three unsuccessful attempts at the original address.

The SF-36 is a 36 item questionnaire which measures eight parameters of health status physical functioning (10 items), role limitations because of emotional problems ( 3 items), role limitations because of physical problems (4 items), social functioning (2 items), mental health ( 5 items), bodily pain ( 2 items), vitality (4 items), and general health perceptions (5 items). ${ }^{1}$ For each parameter, scores are coded, summed, and transformed to a scale from 0 to 100 , with higher scores indicating better health.

Each respondent was also asked whether they had been treated by a doctor for any of the following conditions in the previous 12 months - asthma, diabetes, arthritis or rheumatism, back pain, sciatica, anxiety or stress, depression, problems with sleeping, high blood pressure, and angina. In addition each respondent was asked whether they had ever had a heart attack or stroke.

Mean scores for each of the 8 parameters in the SF-36 were derived for subjects with and 
Mean difference short form 36 questionnaire scores and $95 \%$ confidence intervals (in bracket) for self reported disease groups (corrected for age and $\operatorname{sex}$ )

\begin{tabular}{|c|c|c|c|c|c|c|c|c|}
\hline $\begin{array}{l}\text { Dised } \\
\text { grouf }\end{array}$ & $\begin{array}{l}\text { hysical } \\
\text { inctioning }\end{array}$ & $\begin{array}{l}\text { le- } \\
\text { cotional }\end{array}$ & $\begin{array}{l}\text { ole- } \\
\text { ysical }\end{array}$ & $\begin{array}{l}\text { Social } \\
\text { functioning }\end{array}$ & & & Vitality & $\begin{array}{l}\text { health } \\
n\end{array}$ \\
\hline $\begin{array}{l}\text { sthma } \\
(=55) \\
\text { iabetes } \\
(=23) \\
\text { rthritis } \\
=158) \\
\text { ack pain } \\
=167) \\
\text { ciatica } \\
=52 \text { ) } \\
\text { ypertension } \\
=108 \text { ) } \\
\text { ngina } \\
=61 \text { ) } \\
\text { nxiety } \\
=73 \text { ) } \\
\text { epression } \\
=55) \\
\text { eart attack } \\
=36 \text { ) } \\
\text { (roke } \\
=26 \text { ) }\end{array}$ & $\begin{array}{c}-17 \cdot 8 \\
(-11,-24 \cdot 4) \\
-6 \cdot 3 \\
(3 \cdot 9,-16 \cdot 5) \\
-22 \cdot 9 \\
(-18 \cdot 6,-27) \\
-17 \cdot 2 \\
(-13 \cdot 2,-21) \\
-28 \cdot 3 \\
(-21 \cdot 7,-35) \\
-16 \cdot 7 \\
(-11 \cdot 6,-22) \\
-22 \cdot 6 \\
(-16 \cdot 2,-29) \\
-20 \cdot 5 \\
(-15,-26 \cdot 3) \\
-16.5 \\
(-9 \cdot 8,-23 \cdot 1) \\
-24 \cdot 5 \\
(-16 \cdot 3,-33) \\
-27 \cdot 2 \\
(-17 \cdot 5,-37)\end{array}$ & $\begin{array}{l}-8 \cdot 6 \\
(1 \cdot 0,-18 \cdot 1) \\
-20 \cdot 9 \\
(-6 \cdot 6,-35 \cdot 3) \\
-8 \cdot 8 \\
(-2 \cdot 3,-15 \cdot 2) \\
-11 \cdot 2 \\
(-5 \cdot 3,-17 \cdot 0) \\
-12 \cdot 6 \\
(-2 \cdot 9,-22 \cdot 2) \\
-14 \cdot 1 \\
(-7 \cdot 0,-21 \cdot 5) \\
-20 \cdot 4 \\
(-11 \cdot 2,-30) \\
-46 \cdot 6 \\
(-39,-54 \cdot 4) \\
-47 \cdot 0 \\
(-38,-56) \\
-14 \cdot 8 \\
(-3 \cdot 0,26 \cdot 6) \\
-21 \cdot 2 \\
(-7 \cdot 1,-35)\end{array}$ & $\begin{array}{l}-25 \cdot 4 \\
(-15 \cdot 3,-35) \\
-13 \cdot 3 \\
(2 \cdot 2,-28 \cdot 8) \\
-30 \cdot 8 \\
(-24,-37 \cdot 4) \\
-26 \cdot 7 \\
(-21,-32 \cdot 8) \\
-45 \cdot 0 \\
(-34 \cdot 9,-55) \\
-19 \cdot 0 \\
(-11 \cdot 1,-27) \\
-27 \cdot 1 \\
(-17 \cdot 3,-37) \\
-26 \cdot 6 \\
(-17 \cdot 6,-35) \\
-23 \cdot 8 \\
(-13 \cdot 6,-34) \\
-21 \cdot 5 \\
(-8 \cdot 8,-34 \cdot 3) \\
-14 \cdot 0 \\
(1 \cdot 3,-29)\end{array}$ & $\begin{array}{l}-14 \cdot 0 \\
(-7 \cdot 3,-22 \cdot 4) \\
-11 \cdot 6 \\
(0,-23 \cdot 1) \\
-17 \cdot 5 \\
(-12,-22 \cdot 6) \\
-14 \cdot 7 \\
(-10 \cdot 1,-19) \\
-23 \cdot 3 \\
(-15 \cdot 7,-31) \\
-11 \cdot 8 \\
(-6,-17 \cdot 6) \\
-11 \cdot 0 \\
(-3 \cdot 6,-18 \cdot 4) \\
-29 \cdot 2 \\
(-23,-35 \cdot 7) \\
-30 \cdot 1 \\
(-23,-37 \cdot 4) \\
-15 \cdot 1 \\
(-5 \cdot 6,-24 \cdot 6) \\
-26 \cdot 2 \\
(-16,-36 \cdot 3)\end{array}$ & $\begin{array}{l}-7 \cdot 5 \\
(-2 \cdot 2,-12 \cdot 8) \\
-9 \cdot 3 \\
(-1 \cdot 1,-17 \cdot 5) \\
-6 \cdot 6 \\
(-30,-10 \cdot 2) \\
-7 \cdot 9 \\
(-4 \cdot 7,-11 \cdot 2) \\
-8 \cdot 9 \\
(-3 \cdot 5,-14 \cdot 3) \\
-7 \cdot 1 \\
(-3 \cdot 8,-11 \cdot 2) \\
-8 \cdot 4 \\
(-3 \cdot 3,-13 \cdot 6) \\
-27 \cdot 6 \\
(-23 \cdot 3,-32) \\
-27 \cdot 5 \\
(-23,-33) \\
-6 \cdot 8 \\
(-0 \cdot 1,-13 \cdot 4) \\
-10 \cdot 9 \\
(-3 \cdot 0,-18 \cdot 8)\end{array}$ & $\begin{array}{l}-10 \cdot 2 \\
(-2 \cdot 3,-18 \cdot 2) \\
-0 \cdot 54 \\
(12,-12 \cdot 9) \\
-31 \cdot 9 \\
(-27,-37) \\
-29 \cdot 9 \\
(-25 \cdot 4,-34) \\
-38 \cdot 2 \\
(-30 \cdot 5,-46) \\
-6 \cdot 4 \\
(-0 \cdot 2,-13) \\
-16 \cdot 8 \\
(-9 \cdot 1,-24 \cdot 6) \\
-17 \cdot 4 \\
(-10 \cdot 4,-24) \\
-20 \cdot 3 \\
(-12 \cdot 4,-28) \\
-10 \cdot 4 \\
(-0 \cdot 5,-20 \cdot 3) \\
1 \cdot 3 \\
(13 \cdot 2,-11)\end{array}$ & $\begin{array}{l}-15 \cdot 0 \\
(-8 \cdot 7,-21 \cdot 2) \\
-12 \cdot 7 \\
(-3 \cdot 0,-22 \cdot 5) \\
-17 \cdot 6 \\
(-13 \cdot 5,-22) \\
-14 \cdot 9 \\
(-11 \cdot 1,-19) \\
-17 \cdot 8 \\
(-11 \cdot 4,-24) \\
-10 \cdot 0 \\
(-5 \cdot 2,-15) \\
-18 \cdot 1 \\
(-12,-24 \cdot 2) \\
-23 \cdot 8 \\
(-18 \cdot 4,-29) \\
-24 \cdot 2 \\
(-18 \cdot 1,-30) \\
-18 \cdot 3 \\
(-11,-26 \cdot 1) \\
-8 \cdot 3 \\
(1 \cdot 1,-18)\end{array}$ & $\begin{array}{l}-19 \cdot 5 \\
(-13 \cdot 1,-25 \cdot 9) \\
-15 \cdot 6 \\
(-5 \cdot 7,-25 \cdot 9) \\
-21 \cdot 5 \\
(-17 \cdot 3,-25 \cdot 7) \\
-16 \cdot 2 \\
(-12 \cdot 3,-20 \cdot 1) \\
-23 \cdot 4 \\
(-16 \cdot 9,-29 \cdot 9) \\
-16 \cdot 4 \\
(-11 \cdot 4,-21 \cdot 3) \\
-27 \cdot 2 \\
(-21 \cdot 0,-33 \cdot 3) \\
-27 \cdot 3 \\
(-22,-32 \cdot 8) \\
-22 \cdot 8 \\
(-16.5,-29 \cdot 2) \\
-24 \cdot 0 \\
(-16.0,-32 \cdot 0) \\
-15 \cdot 4 \\
(-5.9,-25 \cdot 0)\end{array}$ \\
\hline
\end{tabular}

without the specific conditions, and the differences were calculated. A negative score signifies poorer health in those with the condition compared with those without. Because the prevalence of conditions and health status parameters were influenced by age and sex, multiple linear regression was used to compare the mean score differences while adjusting for the effects of age and sex. This was carried out by analysis whereby each of the 8 parameters of the SF-36 was separately included in models as the dependent variables and age, sex, and the presence of each disease (separately) were included as the independent variables. Age was included as a continuous variable, and sex and the disease in question as binary categorical variables. The partial regression coefficients, and their standard errors, of the disease variables were used to calculate $95 \%$ confidence intervals for differences between those with and without the particular diseases.

\section{Results}

From the 1201 individuals selected, $192(16 \%)$ were found to have moved or died, and 827 $(69 \%)$ questionnaires were completed. Exclusion of those who had moved or died yielded a corrected response rate of $82 \%$. A total of 55 $(6.6 \%)$ people reported being treated for asthma, $23(2.8 \%)$ for diabetes, $158(19 \cdot 1 \%)$ for arthritis, $167(20 \cdot 2 \%)$ for back pain, $52(6.3 \%)$ for sciatica, $108(13 \cdot 1 \%)$ for high blood pressure, $61(7.4 \%)$ for angina, $73(8.8 \%)$ for anxiety, $55(6.6 \%)$ for depression, and 85 $(10.3 \%)$ for sleeping problems. Thirty six $(4.4 \%)$ people reported having had a heart attack and $26(3.1 \%)$ a stroke. The prevalence of treated cases of angina, diabetes, hypertension, and strokes and heart attacks increased with age $(\mathrm{p}<0.01)$.

The table gives the mean difference in scores for each parameter of the SF-36 in those with and without the specific conditions. All perceived themselves to be in significantly poorer health than those without the condition. Patients with anxiety or depression reported the worst health experience in role limitations from emotional problems and mental health. Patients with back pain, arthritis, or sciatica registered the three highest negative scores in bodily pain and role limitations because of physical problems. The results for those with sleeping disorders have been reported. ${ }^{5}$

\section{Discussion}

This study contains data on the health status of patients suffering from the largest number of disease groups yet reported in published studies, using the SF-36. Garratt et al reported on the health status of 1310 patients suffering from back pain, menorrhagia, suspected peptic ulcer, and varicose veins in Scotland. ${ }^{6}$ Stewart et al reported on the health status of 8517 persons suffering from one of nine conditions using a shorter, earlier version of the Medical Outcomes Study instrument, the SF-20, which contains 20 rather than 36 questions, and 6 rather than 8 parameters. ${ }^{7}$ Because of this the results of this and the latter studies cannot be compared.

Only one condition, back pain, is common to both our study and that reported by Garrett et al. In both studies, patients with back pain scored significantly lower on each of the 8 parameters measured by the SF-36. In all but one of these parameters, however, general health perceptions, the mean score differences in our study were much smaller than in the Scottish study. There is an obvious reason why this should be so. Our study classified people as having back pain if they reported being treated by a doctor for back pain at any time during the previous 12 months, whereas Garratt et al identified patients referred to outpatients or treated by their general practitioners during a three month period. The difference in timescales will mean that more of the patients in our study would be expected to have less active problems thereby resulting in a higher mean score (better health) in parameters such as physical functioning, social functioning, bodily pain, or vitality.

The difference between the results in the two studies could be a function of differences in methodology; self reported, physician treated cases were counted in this study and physician reported cases in the study of Garratt et al. Self 
reported, physician treated illness may not be as accurate as physician reports but is frequently used in classifying patients into diseased and healthy groups, particularly in studies on asthma. ${ }^{8}$ The use of self reported physician diagnosed illness as a case definition also has the advantage that it is suitable for use in a population based study and avoids the selection bias inherent in general practice or hospital based studies. Moreover, although a case definition based on self reported illnesses is unlikely to be $100 \%$ sensitive or specific, the resulting misclassification is likely to diminish, rather than strengthen, the apparent effects of any real associations. Thus, when an association is observed, it is likely to be of significance.

The alternative to using self reported or physician reported illness is the use of specific diagnostic instruments, which is not a perfect solution either. Consider the use of the Rose Questionnaire for angina, the most frequently used community diagnostic measure ${ }^{9}$ The sensitivity and specificity of this instrument has never been measured in a community setting, but assuming the unlikely high figure of $95 \%$ for both, its use in a population with a true prevalence of, say, $7 \%$ would result in an apparent prevalence of $11.65 \%$, and a large degree of misclassification. Apart from questions about the usefulness of established diagnostic questionnaires in relatively low prevalence situations, the inclusion of a large number of such instruments in a community study would be logistically impossible.

In our study the groups of conditions included were chosen on the grounds that they were common conditions and it should be reasonably easy for an individual to determine whether they fitted into any of the categories. Each participant had the option of answering "unsure" to each of the questions on morbidity. In all cases the number reporting an illness outnumbered those "unsure" by at least $6: 1$. In addition, the age distribution of cases was as expected, with the prevalence of diabetes, angina, hypertension, heart attacks, and strokes increasing with age, further supporting the validity of the self reported data. This is further enhanced by the observation that the mean score difference varies in a predictable manner among the disease group - that is, that patients with anxiety or depression reported the worst health experience in role limitations because of emotional problems and mental health and patients with back pain, arthritis, or sciatica registered the three lowest scores in bodily pain and role limitations because of physical problems.

The comparisons between the 11 patient groups in the table are worthy of note. The reader can, at a glance, compare the profiles of the groups and determine the relative impact on the patient of the various diseases. It is possible to compare disease groups such as arthritis, angina, and depression, and gain an impression of the health related quality of life of persons with such conditions. There are two obvious uses for such data. Firstly, they allow health planners to compare the health related quality of life of people suffering from dissimilar conditions. These data, when considered alongside information on the numbers affected, the benefits of therapy, and current disease specific expenditure, would provide useful additional information when strategic decisions on the shifting of resources between treatment groups are being made. Secondly, they provide a baseline of the current health related quality of life of individuals suffering from a variety of conditions. As newer and better treatments are developed and health care purchasing becomes more needs and outcomes based, serial surveys would indicate whether such changes are followed by the expected improvements in the health status of individuals with specific conditions. In this respect there is a need for larger and more comprehensive surveys than this one.

Our results indicate that serial surveys of 3000 respondents would be required to yield an $80 \%$ chance of detecting a 12 points improvement $(50 \%)$ in physical functioning in those with strokes, one of the least common conditions in our survey. The corresponding sample size for those with arthritis, a more prevalent condition, is only 600 .

An alternative approach to increasing the sample size by such an extent is to enrich the sample with more cases by proportionately sampling more elderly people in whom many of the conditions are more common. Yet another approach would be to amalgamate data from adjacent health districts to increase the effective sample size and increase the statistical power at the regional level without increasing the cost per district.

The alternative approach to monitoring health status changes at the population level in those with specific diseases would be to create disease registers for each condition of interest and carry out individual studies into each condition. This approach, however, is likely to be considerably more expensive. Health authorities interested in monitoring the health status of individuals with chronic conditions should consider adopting the population survey approach outlined in this paper.

1 Ware JE, Sherbourne CD. The MOS 36 item short-form health survey (SF-36) 1: Conceptual framework and item selection. Med Care 1992;30:473-80.

2 Ware JE, Brock RH, Williams RN, Stewart AL, DaviesAvery A. Conceptualisation and measurement of health for adults in the health insurance study. Vol. 1 . Model of health adults in the health insurance study. Vol. 1. Modol of health and

3 Brazier JE, Harper R, Jones NMB, O'Cathain A, Thomas KJ, Usherwood T, Westlake L. Validating the SF-36 health survey questionnaire: new outcome measure for primary care. $B M \mathcal{A}$ 1992;305:160-4.

4 Jenkinson C, Coulter A, Wright L. The short form 36 (SF-36) health survey questionnaire: normative data for adults of working age. $B M F$ 1993;306:1437-40.

5 Lyons RA, Fielder H, Littlepage BNC. SF-36 and sleep disorders. BMf 1993;307:449.

6 Garratt AM, Ruta DA, Abdalla MI, Buckingham JK, Russell IT. The SF-36 health survey questionnaire: an outcome measure suitable for routine use within the NHS? BMF 1993;306:1440-4.

7 Stewart A, Greenfield S, Hays RD, Wells K, et al. Functional status and wellbeing of patients with chronic conditions. status and wellbeing of 13 .

8 Dodge $\mathrm{RR}$, Burrows $\mathrm{B}$. The prevalence and incidence of asthma and asthma-like symptoms in a general population asthma and asthma-like symptoms in a gener.

9 sample. Am Rev Respir Dis 1980;122:567-75. R. Cardiovascular survey methods. 2nd ed. Geneva: World Health vascular survey methods. 